\title{
A Dynamic Role of the Medial Temporal Lobe during Retrieval of Declarative Memory in Man
}

\author{
Karl Magnus Petersson,* Christina Elfgren, $†$ and Martin Ingvar*,1 \\ *Cognitive Neurophysi ol ogy R2-01, Department of Clinical Neuroscience, Karolinska Hospital, 171 76 Stockholm, Sweden; \\ and †Department of Psychogeriatrics, University Hospital of Lund, 22209 Lund, Sweden
}

Received February 12, 1997

\begin{abstract}
Understanding the role of the medial temporal lobe (MTL) in learning and memory is an important problem in cognitive neuroscience. Memory and learning processes that depend on the function of the MTL and related diencephalic structures (e.g., the anterior and mediodorsal thalamic nuclei) are defined as declarative. We have studied the MTL activity as indicated by regional cerebral blood flow with positron emission tomography and statistical parametric mapping during recall of abstract designs in a less practiced memory state as well as in a well-practiced (well-encoded) memory state. The results showed an increased activity of the MTL bilaterally (including parahippocampal gyrus extending into hippocampus proper, as well as anterior lingual and anterior fusiform gyri) during retrieval in the less practiced memory state compared to the well-practiced memory state, indicating a dynamic role of the MTL in retrieval during the learning processes. The results also showed that the activation of the MTL decreases as the subjects learn to draw abstract designs from memory, indicating a changing role of the MTL during recall in the earlier stages of acquisition compared to the well-encoded declarative memory state. $\odot 1997$ Academic Press
\end{abstract}

\section{INTRODUCTION}

Learning processes and memory are fundamental brain functions, enabling the brain to learn and adapt in a changing environment. Learning can be defined as processes by which the central nervous system functionally restructures its processing pathways or its representations of information. Memory can then be viewed as the resulting changes in the processing pathways or representations of information due to these learning processes. From a parallel distributed processing perspective (Amit, 1989, 1995; Arbib, 1995; Haykin, 1994; Hertz et al., 1991; Rumelhart and McClelland, 1986),

\footnotetext{
1 To whom correspondence should be addressed.
}

learning in a neural network is a dynamical consequence of information processing and network plasticity. Memory consists of changes in the synaptic structure of the processing system, i.e., its functional (or effective) connectivity. Such changes can be detected indirectly at a behavioral level as changes in the performance of a task, as a result of learning or practice.

Understanding the role of the medial temporal lobe (MTL) in learning and memory is an important problem in cognitive neuroscience (Schacter et al., 1996). Memory and learning processes that depend on the function of the MTL and related diencephalic structures (e.g., the anterior and mediodorsal thalamic nuclei) are defined as declarative (Squire, 1994; Squire et al., 1993; Squire and Knowlton, 1995). Studies of human amnesia and nonhuman primate models of human amnesia have identified anatomical and functional components of the declarative memory system in the MTL. These consist of the hippocampus and adjacent, anatomically related cortex, including the parahippocampal, entorhinal, and perirhinal cortices (Markowitsch, 1995; Squire and Zola-Morgan, 1991; ZolaMorgan and Squire, 1993). The MTL has widespread and reciprocal connections with associative neocortex as well as subcortical structures and is essential for establishing long-term declarative memories of facts or general knowledge (semantic memory) and events (episodic memory) (Insausti et al., 1987a,b; Schacter and Tulving, 1994; Squire et al., 1993; Suzuki and Amaral, 1994; Tulving, 1995; Zola-Morgan and Squire, 1993).

The MTL has been ascribed different functions; for example, it may be needed to bind together the distributed representations contained within different association areas in the neocortex that represent the declarative memory as a whole (conjunctive learning), to enable the rapid acquisition of declarative knowledge for long-term integrative learning, the creation of flexible cognitive map representations (or relational representations), reducing interference (or negative transfer) as well as representation separation, and memory 
consolidation (Bibbig et al., 1995; Brown, 1990; Eichenbaum, 1994; McClelland, 1994; McClelland and Goddard, 1996; McClelland et al., 1995; Mesulam, 1990; Nadel, 1994; O'Reilly and McClelland, 1994; Shapiro and Olton, 1994; Squire, 1992; Squire and ZolaMorgan, 1991; Treves and Rolls, 1994). Distributed activity in the neocortex is thought to be the substrate for perception and short-term memory (Damasio and Damasio, 1994; Fuster, 1994; Mesulam, 1990; Singer and Gray, 1995). If such distributed cortical activity is to be stored as a long-term declarative memory, then the MTL structures must be engaged at the time of learning (Squire, 1992; Squire and Zola-Morgan, 1991) to bind together the distributed representations. Presumably, the final storage site of declarative knowledge is in the neocortex, i.e., the neocortical memory network (Eichenbaum, 1994; Fuster, 1994; McClelland et al., 1995; Mesulam, 1990; Nadel, 1994; Squire and Alvarez, 1995; Squire and Knowlton, 1995; Squire and Zola-Morgan, 1991; Treves and Rolls, 1994; ZolaMorgan and Squire, 1993). This implies that declarative learning and memory storage is dependent on some type of interaction between the MTL and the neocortex.

The phenomenon of temporally graded retrograde amnesia has given rise to the concept of memory consolidation, meaning the high-level process by which declarative memory becomes independent of the MTL memory system (Squire, 1992; Squire and Alvarez, 1995; Squire et al., 1993) [this concept of memory consolidation should be carefully distinguished from other concepts of memory consolidation, for example with biochemical connotations (Squire and Alvarez, 1995)]. It has been hypothesized that repeated reactivations of the neocortical representations of declarative memories would strengthen the neocortical interconnections so that eventually the neocortical memory network can support declarative memory retrieval independently of the MTL (Alvarez and Squire, 1994; McClelland, 1994; McClelland and Goddard, 1996; McClelland et al., 1995; Squire and Alvarez, 1995; Treves and Rolls, 1994). This suggest that the interaction between the MTL and the neocortex may be dynamic, i.e., changing as a function of repeated activations of the interacting networks or as a dynamical consequence of repeated processing and network plasticity.

J ones-Gotman has devised a free recall abstractdesign-list-learning paradigm sensitive to MTL lesions (J ones-Gotman, 1986). In order to explore the functional aspects of the MTL, we modified J onesGotman's test and constructed a PET paradigm to test recall of abstract visuospatial designs, in a less practiced memory state (novel recall, NR) and in a wellpracticed (well-encoded) memory state (trained recall, TR). We hypothesized that the interaction between the MTL and the neocortex during the recall of the abstract design would change as a result of practice. Presumably practice (in this case repeated encoding and recall) would repeatedly reactivate the neocortical representations and hence strengthen interconnections in the neocortical network in such a way that the neocortex eventually can support declarative retrieval less dependent on the interaction with the MTL. We also hypothesized that this change would be detectable as a difference in the activity of the MTL during NR compared to TR.

\section{MATERIALS AND METHODS}

\section{Subjects}

Eleven right-handed [Edinburgh handedness inventory (Oldfield, 1971; Raczkowski \&t al., 1974)] healthy male subjects (mean age 24, 22-29) were included in the study. The subjects were prescreened and none of the subjects used any medication or had a history of drug abuse (including nicotine), head trauma, neurol ogical or psychiatric illness, or had a history or a family history of neurological or psychiatric illness. All had 1-5 years of university level education. The study was approved by the local Ethics and Radiation Safety Committees at the Karolinska Hospital. I nformed consent was given by all subjects.

\section{The Experimental Paradigm and Procedures}

The subjects practiced all aspects of the experimental paradigm (with sham injections) for approximately 20 min in the PET camera before the experiment started, in order to get accustomed to the various aspects of the paradigm.

The experimental paradigm consisted of two identical blocks separated by approximately $30 \mathrm{~min}$ of rest when the subjects were allowed to leave the PET camera (Fig. 1C). Within each block scanning was done during reference state (RS), NR, and TR. Each block consisted of six scans, with at least $10 \mathrm{~min}$ between scans, in the order RS/NR/NR/training period/TR/TR/RS.

In the RS the subjects filled in the contours of simple predrawn designs [of the same size as the abstract designs (see below), such as squares, circles, and triangles]. These were shown on the monitor (Fig. 1a). Visual feedback of the hand was provided by closedcircuit TV and shown on a monitor just below the computer screen (Fig. 1a). Following the first RS scan in a block, the subject was engaged in the basic experimental cycle (encoding-recall cycle) which consisted of an encoding part and a recall part (Fig. 1b). During encoding (copying), a list of 15 separate abstract designs, simple enough to be copied rapidly but sufficiently complex to discourage direct descriptive naming (J ones-Gotman, 1986), were shown for $15 \mathrm{~s}$ each on a computer screen (Fig. 1a). The subject copied 

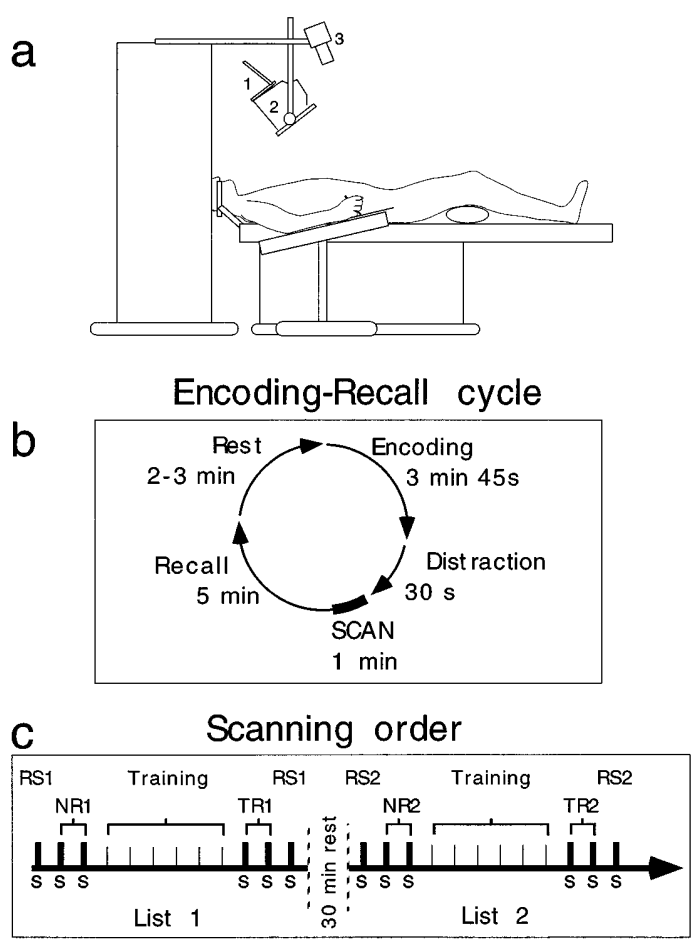

FIG. 1. (a) The experimental PET camera setup. 1, computer screen for presentation of the abstract designs to be copied during encoding; 2, monitor for closed-circuit visual feedback of the hand; and 3, camera feeding into the monitor in the closed-circuit visual feedback. (b) Encoding-recall cycle. During encoding each of the 15 abstract designs were copied one time. Then a distractor text was read for $30 \mathrm{~s}$. Finally, during recall the designs were reproduced as faithfully as possible. (c) The scanning order of the 12 scans: 2 RS, 2 $N R$, and 2 TR scans in each block (bold tics; s, scanning) and the training period (approximately $30 \mathrm{~min}$ ) of six encoding-recall cycles in each block (thin tics). Different lists were used for the two blocks.

each design one time, and all designs were copied with a pen on the same paper. Following encoding the subject read a nonsense text (shown on the computer screen) al oud for $30 \mathrm{~s}$ in order to prevent recency effects (Baddeley, 1995). After this the recall part was started, the bolus injection given (the PET scanning started as soon as the bolus reached the brain and continued for the next $60 \mathrm{~s}$ ), and the subject was instructed to start drawing the designs from memory. The subject reproduced the designs as faithfully as possible in any order during recall. The subject had $5 \mathrm{~min}$ at his disposal to reproduce the designs in order not to feel time as a limiting factor. This would also allow the subject to fully report what had been retained in long-term memory.

Two different lists of 15 designs were used, one for each block, balanced over blocks. During each block there was a training period between the second NR scan and the first TR scan (Fig. 1c). The training period consisted of 6 encoding-recall cycles. Altogether the subject went through 10 encoding-recall cycles and was scanned on the first two and the last two recall procedures during each block. The time between when the subject was first confronted with the list of abstract designs and the last time during trained recall was approximately $1 \mathrm{~h}$ and $15 \mathrm{~min}$, for each list.

The instructions to the subject were to copy each of the 15 designs one time as faithfully as possible, then to read the text aloud, and finally, during the recall part (scanning), to reproduce the designs as faithfully as possible in any order. These instructions were repeated before every new encoding-recall cycle (when scanning was to be done) started.

During the experiment the subjects hand movements were confined to $\approx 12 \times 18 \mathrm{~cm}$ by the field of view of the monitor $\left(\approx 5^{\circ}\right)$.

\section{PET Scanning}

Each subject underwent 12 measurements of re gional cerebral blood flow (rCBF) with a 3D Ecat Exact HR PET scanner and bolus injections of [150]butanol (Berridge et al., 1990; Wienhard et al ., 1994) (for details see Ingvar et al., 1994). The camera was used in 3D-sampling mode producing 60-s tracer uptake images. The different tasks were started at the time of tracer injection and the scanning was automatically initiated when the activity level in the brain exceeded a predetermined level above background. Scatter correction was done and a 2D-transmission scan was used for attenuation correction.

\section{Data Analysis}

The PET images were realigned, spatially normalized, and transformed into a common stereotactic space (Talairach and Tournoux, 1988), 3D-Gaussian filtered (14-mm FWHM), proportionally scaled to account for global confounders and analyzed with statistical parametric mapping (SPM) (Friston et al ., 1995a,b; Worsley et al., 1992). The order of scans within each state was used as a confounding covariate. Since a regional hypothesis concerning the role of the medial temporal lobe in declarative learning and memory function was made, the search volume was restricted to $z$ coordinates between +8 and -28 in the Talairach space. To test hypotheses about regionally specific condition or covariate effects, estimates were compared using linear contrasts. The resulting set of voxel values for each contrast constitutes a statistical parametric map of the t statistic, SPM(t), that was transformed to standard normal distributed parameters, SPM(Z), and thresholded at 3.09 (omnibus significance $P<0.001$ ). The resulting activated regions were then characterized in terms of spatial extent $(\mathrm{k})$ and peak-height $(\mathrm{u})$ of local maxima. All reported $P$ values are corrected for multiple nonindependent comparisons.

The reproduced designs were scored (the recall score) according to the following: near exact reproduction of 




FIG. 2. The centers of the spherical regions of interest were chosen according to the Talairach-Tornoux space and were located in hippocampus proper (approximately $[x, y, z]=[ \pm 31,-29,-8] \pm 1 \mathrm{~mm}$ ) and in parahippocampal gyrus (approximately $[x, y, z]=$ $[ \pm 29,-23,-20] \pm 1 \mathrm{~mm}$ ) with the hel $p$ of an average MR brain (10 normal subjects) representing the Talairach-Tornoux space in SPM 95 and the Karolinska CBA (Greitz et al., 1991).

the design $=3$; close reproduction with one addition, distortion, omission, inversion, or rotation of a detail = 2; fair reproduction that contained two of the abovementioned mistakes $=1$; and anything worse was given 0 .

Regions of interest (ROI) analysis was done to further analyze the response in different parts of the MTL. The ROI s consisted of spherical vol umes. The centers of the ROIs (see Fig. 2) were chosen according to the Talairach-Tornoux spaceand were located in hippocampus proper (approximately $[x, y, z]=[ \pm 31,-29,-8] \pm$ $1 \mathrm{~mm}$ ) and in parahippocampal gyrus (approximately $[x, y, z]=[ \pm 29,-23,-20] \pm 1 \mathrm{~mm})$ with the help of an average MR brain (10 normal subjects) representing the Talairach-Tornoux space in SPM 95 and the Karolinska computerized brain atlas (CBA) (Greitz et al., 1991). The locations of the ROIs were also chosen so that the distance between the centers of ROIs in the same hemisphere were approximately $14 \mathrm{~mm}$ apart to minimize mixing caused by the 3D-Gaussian filtering (14-mm FWHM), and the diameters of the ROI s $(6 \mathrm{~mm})$ were chosen so that a given ROI was located inside a given structure. The ROI data were generated with the
CBA computer software from adjusted SPM data and analyzed with a one-factor ANOVA, and specific contrasts were used to test for differences in ROI means between states (i.e., RS, NR, and TR).

\section{RESULTS}

In the given context activation and deactivation are used as synonyms for increased and decreased, respectively, rCBF. The tables of local maxima use the Talairach-Tournoux anatomical designations for anatomy ${ }^{2}$ (Talairach and Tournoux, 1988). All reported $P$ values are corrected for multiple nonindependent comparisons.

\section{Comparing NR with TR}

Our data (Table 1, Fig. 3) show that theactivity in the MTL is significantly higher during novel recall, when

\footnotetext{
2 Because of the regionally specific hypothesis made, we concentrate on the results relating to the medial temporal lobe. Other results will be reported elsewhere.
} 


\section{TABLE 1}

Local Maxima of Significant Activation When Comparing Novel Recall to Trained Recall Listed as Local Z Maxima

\begin{tabular}{cccc}
\hline Region & $\begin{array}{c}\text { Brodmann } \\
\text { area }\end{array}$ & {$[x, y, z]^{a}$} & $Z$ \\
score $P\left(Z_{\max }>u\right)^{b}$ \\
\hline
\end{tabular}

(a) Right medial and inferotemporal region: 1002 voxels, $\mathrm{P}<0.001$

Parahippocampal gyrus

Parahippocampal gyrus

Fusiform gyrus

Fusiform gyrus

Inferior temporal gyrus

BA 36

$30-28-20 \quad 5.66$

0.000

BA $28 / 35$

BA $36 / 37$

$\begin{array}{llll}22 & -14 & -28 & 4.73\end{array}$

$\begin{array}{llll}36 & -40 & -12 & 4.72\end{array}$

$\begin{array}{llll}50 & -48 & -16 & 4.90\end{array}$

BA 37

$\begin{array}{llll}50 & -64 & -20 & 4.87\end{array}$

0.001

0.002

0.001

0.001

(b) Left medial and inferotemporal region: 1521 voxels, $\mathrm{P}<0.001$

Parahippocampal/

fusiform gyrus

Fusiform gyrus

Fusiform gyrus

Inferior temporal

gyrus

BA 36

BA 36

BA 37

$\begin{array}{llll}-34 & -26 & -24 & 5.97\end{array}$

$\begin{array}{llll}-34 & -38 & -16 & 5.79\end{array}$

$\begin{array}{llll}-44 & -58 & -8 & 5.66\end{array}$

0.000

0.000

0.000

$\begin{array}{llllll}\text { BA } 20 / 37 & -58 & -40 & -12 & 4.49 & 0.004\end{array}$

a The coordinates of the local maxima refer to the anatomical space of Talairach and Tournoux (1988).

${ }^{b}$ All $\mathrm{P}$ values are corrected for multiple nonindependent comparisons.

mean performance was $26 \pm 10$ (mean \pm SD, maximum obtainable recall score $=45=15 \times 3$ ), compared to trained recall, when performance was improved to almost perfection ( $44 \pm 1$, improvement $P<0.0001$, Wilcoxon signed rank test). During the approximately $80 \mathrm{~s}$ from bolus injection to the end of scanning, the subjects reproduced $6.2( \pm 1.3)$ designs during NR and $9.3( \pm 2.5)$ during TR. The bilaterally activated MTL inferotemporal regions (volume of right 1002 voxels, $P=0.001$, and volume of left 1521 voxels, $P<0.001$ ) included parahippocampal gyrus extending into hippocampus proper, as well as anterior lingual, anterior fusiform, and inferior temporal gyri according to the Karolinska computerized brain atlas (Greitz et al., 1991). The local maxima are given in Table 1.

We also correlated rCBF with the mean learning curve (i.e., using the mean recall scores for the different NR and TR scans as a linear contrast) and found negative correlations in the same regions of MTL (Table 2).

\section{Comparing NR with RS}

To see how the relevant MTL inferotemporal regions responded in NR and TR compared to RS we masked with the significant volumes in the NR-TR comparison (thresholding at $P=0.001$ ).

Comparing NR with RS, the same MTL inferotemporal regions (including bilateral parahippocampal gyri extending into right hippocampus proper and lingual, fusiform, and inferior temporal gyri bilaterally, accord- ing to the Karolinska computerized brain atlas) were significantly activated (volume of right MTL inferotemporal region 815 voxels, $P=0.002$, and volume of left MTL inferotemporal region 695 voxels, $P<0.003$ ). There were local activation maxima in the right parahippocampal gyrus (BA 28/35/36, [x, y, z] $=[28,-24$, $-24], Z=4.58, P=0.003)$, right fusiform gyrus/ cerebellum (BA 36/37/20, $[x, y, z]=[30,-38,-24], Z=$ $6.73, P<0.001$ ), right inferior temporal gyrus (BA 37, $[x, y, z]=[48,-62,-20], z=9.60, P<0.001)$, and left fusiform/cerebellum gyrus (BA $37,[x, y, z]=[32,-44$, $-24], Z=6.73, P<0.001)$. There were no significant deactivations in this comparison.

\section{Comparing T R with RS}

Comparing TR with RS, we found a significantly activated right infero-occipitotemporal region (volume $322, \mathrm{P}=0.02$, including right inferior temporal, fusiform, and lingual gyri according to the CBA) and there were local activation maxima in right fusiform gyrus/ cerebellum (BA 36/37/20, [x, y, z] $=[30,-38,-24], \mathrm{Z}=$ $3.82, P<0.05$, and BA 19, $[x, y, z]=[42,-64,-20]$, $Z=6.42, P<0.001$ ). We also found a significantly deactivated left infero-occipitotemporal region (volume $388, \mathrm{P}=0.01$, including left inferior temporal extending into middle temporal, fusiform, and lingual gyri according to the CBA). There were local deactivation maxima in left inferior temporal gyrus (BA 20/37, $[x, y, z]=[-56,-42,-8], Z=6.59, P<0.001)$, left inferior/middle temporal gyrus (BA 21/37, [x, y, z] = $[-48,-54,0], Z=5.65, P<0.001)$, left fusiform gyrus (BA 20, [x,y, z] $=[40,-24,-24], Z=5.02, P<0.001$ ). Masking with the significant volumes in the NR-TR comparison, thresholding at $\mathrm{P}=0.01$, the right inferotemporal region extended into the left MTL (for example, the voxel $[x, y, z]=[-32,-24,-16], z=3.27$, $\mathrm{P}<0.0005$, uncorrected, see Fig. 3).

\section{Results from the RO I Analysis}

The results from the ROI analysis are shown in Table 3. All $P$ values are Bonferroni-corrected for multiple comparisons. The rCBF (arbitrary units, globally normalized to 50) was higher in NR compared to TR in right parahippocampal gyrus (NR 63.7, TR 61.5, P < 0.0005 ), left parahippocampal gyrus (NR 60.6, TR 58.4, $P<0.0005$ ), right hippocampus (NR 62.3, TR 61.1, $P=0.0008$ ), and left hippocampus (NR 63.3, TR 61.9, $P<0.0005)$. The rCBF was higher in NR compared to RS in right parahippocampal gyrus (NR 63.7, RS 61.9, $P<0.0005$ ), left parahippocampal gyrus (NR 60.6, RS 59.2, $P<0.0005$ ), right hippocampus (NR 62.3, RS 60.8, $P<0.0005$ ), and left hippocampus (NR 63.3, RS 62.9, NS). Finally, the rCBF was lower in TR compared to RS in left hippocampus (TR 61.9, RS 62.9, $P=0.001$ ) and left parahippocampal gyrus (TR 58.4, RS 59.2, $P=0.001$ ). 


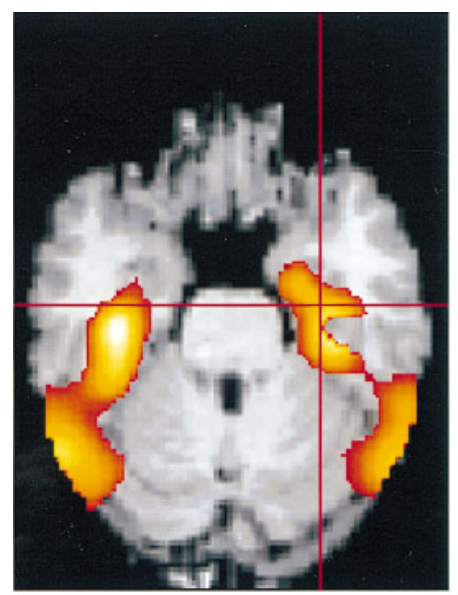

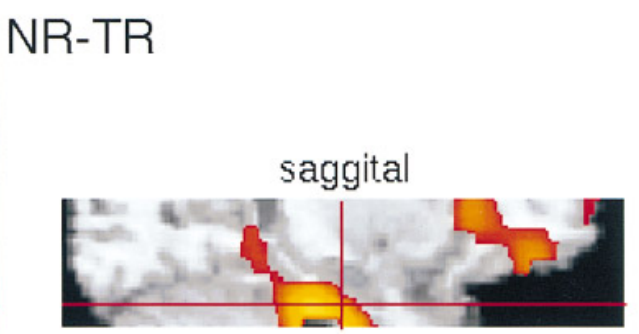

coronal
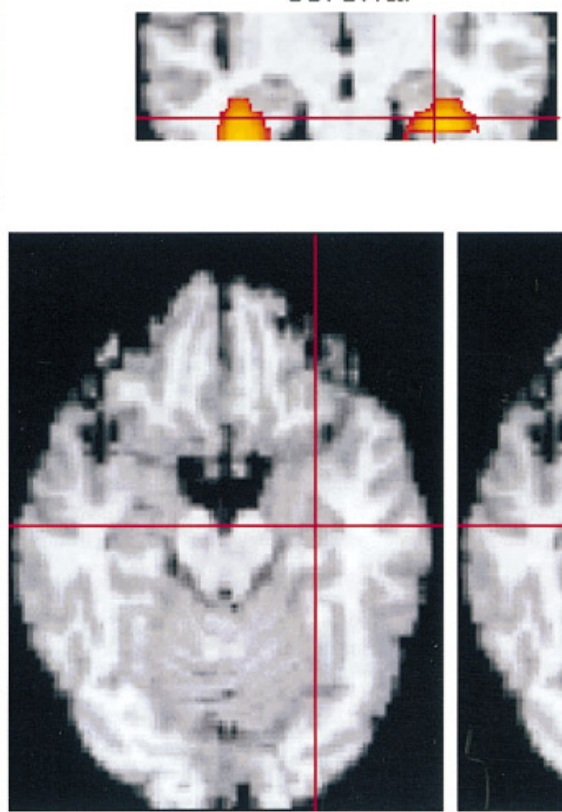

RS-NR

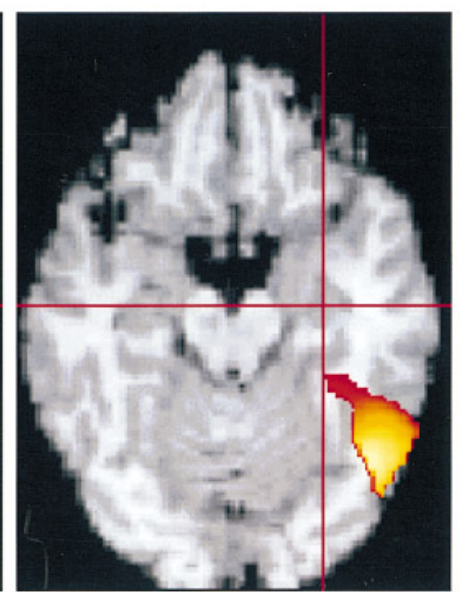

TR-RS
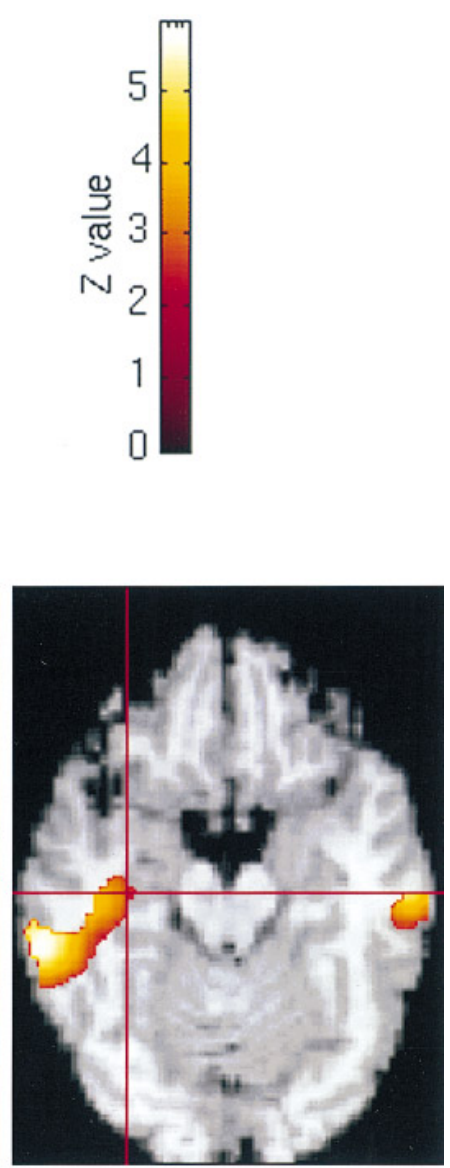

RS-TR

FIG. 3. Relevant regions of activation and deactivation from the different contrasts. Horizontal, saggital, and coronal views of the activated region in NR compared to TR are shown in the first row. This volume of interest was used to mask the other contrast to investigate how this volume of interest responded in the different states (i.e., RS, NR, and TR) shown in the second row. The cross hairs indicate the coordinate $[x, y, z]=[26,-20,-22]$ in the NR-TR and NR-RS contrasts, the coordinate $[x, y, z]=[26,-20,-16]$ in the RS-NR and TR-RS contrasts, and the coordinated $[x, y, z]=[-32,-24,-16]$ in the RS-TR contrast. All contrasts are thresholded at the significance level P $=$ 0.01 for illustrative purposes.

\section{DISCUSSION}

We demonstrate a significantly increased activity in the MTL during the less practiced NR state compared to the well-practiced memory state TR (Fig. 3, Table 1). There were also neocortical differences between NR and TR, including increased activity in prefrontal, anterior insular, anterior cingulate, and parietooccipital, as well as decreased activity including posterior occipitotemporal and visual areas (these results will be reported elsewhere). This is consistent with our hypothesis that the interaction between the MTL and the neocortex would change as a result of repeated encoding and recall.

Medial temporal lobe activations have been reported in a number of memory-related PET studies (e.g., Grady et al., 1995; Grasby et al., 1993; Haxby et al., 1996; Kapur et al., 1995; Schacter et al., 1996). Consistent with this, in NR compared to RS, we find in the
SPM analysis significantly activated MTL inferotemporal regions (including right hippocampus proper and parahippocampal gyri bilaterally). There was a significant local maximum in the right parahippocampal gyrus, and with the more sensitive ROI analysis, increased activity was also detected in the left parahippocampal gyrus (Table 3). This is also consistent with the reported sensitivity of the J ones-Gotman test for MTL lesions, in particular right MTL lesions (J onesGotman, 1986).

Comparing TR with RS we find a significantly activated right infero-occipitotemporal region while we find a significantly deactivated left infero-occipitotemporal region. The different response patterns in the right and the left infero-occipitotemporal region compared with the MTL indicate that thefunctional significance of the infero-occipitotemporal regions is different from that of the medial temporal lobes. 
According to the ROI analysis the activity in the left hippocampus was significantly greater during RS compared to TR (Table 3). Hence, only the left hippocampus is activated in both RS and NR compared to TR. This may reflect that there is an episodic encoding component in RS. This is also consistent with the hypothesis that the left hippocampal formation is more continuously active than the right (Rugg et al., 1996) and therefore more difficult to activate differentially.

Most importantly we demonstrate a significantly increased activity in the MTL during the less practiced NR state compared to the practiced memory state TR (Fig. 3, Table 1). This is consistent with electrophysiological evidence of changing neuronal responses in the hippocampus during conditional spatial responselearning (Cahusac et al., 1993). It is worth stressing that all subjects were fully occupied with declarative recall during the whole scanning time in all the NR and TR scans. It is also worth pointing out that this response pattern in the MTL (i.e., the MTL is more active during NR compared to TR) was repeated for each block in the experiment (i.e., each list of abstract designs, see Fig. 4). Further, we show that the activation of the MTL decreases as the subjects learn to draw abstract designs from memory (Table 2).

There are several possible interpretations of these findings, not necessarily incompatible with one another. It has been hypothesized that memory representations correspond to stable or quasistable dynamical attractors (in a dynamical systems sense; see for ex-

\section{TABLE 2}

Local Maxima of Significant Negative Correlation between Mean Adjusted rCBF and Mean Learning Curve, i.e., Using the Mean Recall Scores for the NR and TR Scans as a Linear Contrast

\begin{tabular}{|c|c|c|c|}
\hline Region & $\begin{array}{l}\text { Brodmann } \\
\text { area }\end{array}$ & {$[x, y, z]^{a}$} & $\begin{array}{l}\mathrm{Z} \\
\text { score } \mathrm{P}\left(\mathrm{Z}_{\max }>\mathrm{u}\right)^{\mathrm{b}}\end{array}$ \\
\hline
\end{tabular}

(a) Right medial and inferotemporal region: 1117 voxels, $\mathrm{P}<0.001$

Parahippocampal gyrus

$\begin{array}{llllll}\text { BA } 36 & 32 & -28 & -20 & 5.54 & 0.000\end{array}$

Parahippocampal gyrus

$\begin{array}{lllll}\text { BA } 28 & 20 & -10 & -28 & 4.18\end{array}$

0.02

Fusiform gyrus

BA $36 / 37$

$\begin{array}{llll}40 & -40 & -12 & 5.49\end{array}$

0.000

Fusiform gyrus

BA 37

$\begin{array}{llll}50 & -46 & -16 & 5.47\end{array}$

0.000

(b) Left medial and inferotemporal region: 1538 voxels, $\mathrm{P}<0.001$

Parahippocampal/

$\begin{array}{llllllll}\text { fusiform gyrus } & \text { BA } 36 & -34 & -26 & -24 & 5.84 & 0.000\end{array}$

$\begin{array}{llllllll}\text { Fusiform gyrus } & \text { BA } 37 & -44 & -58 & -12 & 6.47 & 0.000\end{array}$

Inferior temporal

gyrus

BA 37

$\begin{array}{llll}-58 & -44 & -16 & 5.29\end{array}$

0.004

a The coordinates of the local maxima refer to the anatomical space of Talairach and Tournoux (1988).

${ }^{b}$ All P values are corrected for multiple nonindependent comparisons.
TABLE 3

Results from the ROI Analysis

\begin{tabular}{|c|c|c|c|c|}
\hline State & $\begin{array}{l}\text { ROI } \\
\text { mean }\end{array}$ & $\mathrm{SD}$ & F score & P value \\
\hline
\end{tabular}

(a) Right hippocampus, center of spherical ROI $[x, y, z]=$ $[31,-29,-8]^{a}$

$\begin{array}{lcccc}\text { RS } & 60.8 & 1.6 & & \\ \text { NR } & 62.3 & 1.4 & & \\ \text { TR } & 61.1 & 1.4 & & <0.0005 \\ \text { NR vs RS } & & & 21.0 & \text { NS } \\ \text { TR vS RS } & & & 0.5 & 0.0008 \\ \text { NR vs TR } & & & 15.3 & \end{array}$

(b) Left hippocampus, center of spherical ROI $[x, y, z]=$ $[-31,-29,-8]^{a}$

\begin{tabular}{|c|c|c|c|}
\hline $\mathrm{RS}$ & 62.9 & 1.4 & \\
\hline NR & 63.3 & 1.3 & \\
\hline $\mathrm{TR}$ & 61.9 & 1.1 & \\
\hline NR vs RS & & 2.4 & NS \\
\hline TR vs RS & & 13.8 & 0.001 \\
\hline NR vs TR & & 27.2 & $<0.0005$ \\
\hline
\end{tabular}

(c) Right parahippocampal gyrus, center of spherical ROI $[x, y, z]=[29,-23,-20]^{a}$

$\begin{array}{lrrrr}\text { RS } & 61.9 & 1.5 & & \\ \text { NR } & 63.7 & 1.8 & & \\ \text { TR } & 61.5 & 1.8 & & \\ \text { NR vs RS } & & & 24.0 & <0.0005 \\ \text { TR vS RS } & & & 0.5 & \text { NS } \\ \text { NR vs TR } & & & 15.3 & <0.0005\end{array}$

(d) Left parahippocampal gyrus, center of spherical ROI

$[x, y, z]=[-29,-23,-20]^{a}$

\begin{tabular}{lrrrc} 
RS & 59.2 & 1.6 & & \\
NR & 60.6 & 1.6 & & \\
TR & 58.4 & 1.7 & & $<0.0005$ \\
NR vs RS & & & 15.4 & 0.001 \\
TR vs RS & & & 5.1 & $<0.0005$ \\
NR vs TR & & & 38.0 & $<$ \\
\hline
\end{tabular}

\footnotetext{
a The coordinates refer to the anatomical space of Talairach and Tournoux (1988).

${ }^{b}$ All $\mathrm{P}$ values are Bonferroni-corrected and relate to the significance of difference between the means indicated (SD, standard deviation; NS, nonsignificant, i.e., $\mathrm{P}>0.05$ ).
}

ampleAmit, 1989, 1995; Arbib, 1995; Beck and Schlögl, 1993; Haykin, 1994; Hertz et al., 1991). From a dynamical systems perspective, the low mean NR recall scores may be interpreted as indicating that not all of the neocortical representations (patterns of activation) of the different abstract designs are stable enough to be retrieved successfully (i.e., representing stable or quasistable dynamical attractors). The increased activation of the MTL during NR may then be interpreted as indicating that the initial stabilization of the memory representations are dependent on the MTL-neocortical interaction and therapid plasticity of the MTL. Presumably, repeated encoding and recall would reactivate the neocortical representations and hence strengthen the neocortical interconnections in such a way that the 


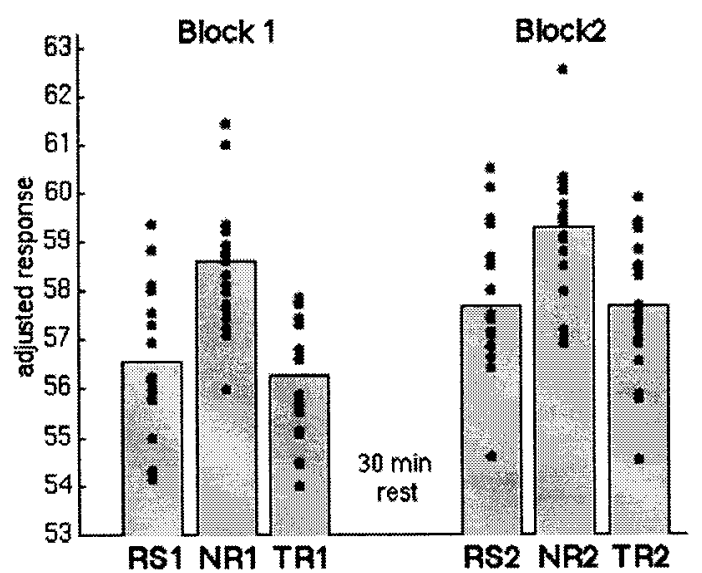

FIG. 4. The adjusted rCBFs in the right parahippocampal gyrus $([x, y, z]=[30,-28,-20])$ are plotted in the diagram since they vary over the different states in the two blocks of scans.

neocortical network eventually can support declarative retrieval, less dependent on the interaction with the $M T L$, as indicated by the decreased activation of the MTL compared to NR. The repeated reactivations may also lead to increased stability and probability of retrieval success as indicated by the increase in the recall scores. This interpretation is also consistent with several recent theoretical hypotheses concerning the role of the MTL (Alvarez and Squire, 1994; McClelland, 1994; McClelland and Goddard, 1996; McClelland et al., 1995; Robins, 1996; Treves and Rolls, 1994).

The changing role of the MTL as a result of practice can also be interpreted in the light of the concept of memory consolidation, meaning the high-level process by which declarative memory representations become independent of the MTL, i.e., as time passes after learning there is a gradual reorganization of declarative memory whereby memories which are initially dependent on the MTL become independent of this system (Squire, 1992; Squire and Alvarez, 1995; Squire et al., 1993). The concept of memory consolidation has its roots in the observation of temporally graded retrograde amnesia, observed in humans, nonhuman primates, and rodents (Squire, 1992). Also, lesions in MTL affect consolidation of recently formed memories but have no effect on consolidated memories (Zola-Morgan and Squire, 1990). The temporal gradient observed in temporally graded retrograde amnesia has been estimated to extend for months to years in humans and, in animal models of temporally graded retrograde amnesia, weeks to months in nonhuman primates and days in rodents (Squire, 1992; Squire and Alvarez, 1995; Zola-Morgan and Squire, 1990). Further, the time course of consolidation will vary not only depending on the species, but also depending on the strength of initial learning and the rate of forgetting (Squire et al., 1993). In our experiment, the time between when the subject was first confronted with the list of abstract designs and the last time during trained recall was approximately $1 \mathrm{~h}$ and $15 \mathrm{~min}$ for each list and the subject was subjected to intense learning during this time.

Memory consolidation is most likely not a unitary neurobiological process, but is subserved by several different neurobiological processes with different time constants. The results of Brasher-Krug et al. (1996) seem to indicate that effects of consolidation in the neocortex are measurable within at most $4 \mathrm{~h}$ at the behavioral level. Presumably this reflects a consolidation processes that started at the time of learning. This would indicate that it may be possible to detect consolidation effects earlier using functional imaging modalities like PET. Practice-related changes in the cortical functional anatomy have been detected with PET within 15 min to $2 \mathrm{~h}$, in sequence learning (F riston et al., 1992; Grafton et al., 1995), in trial-and-error learning of a motor sequence with performance feedback (J enkins et al., 1994), in procedural perceptuomotor learning (Grafton et al., 1992), and in a nonmotor verbalassociative task (Raichle et al., 1994).

There may be a problem with the consolidation interpretation since consolidation has been related to the temporal dimension, and in our experiment this is confounded with practice. However, it has been hypothesized that it is not only time per se that is important for memory consolidation, but also repeated activations of the neocortical representations as a consequence of, for example, new learning events, rehearsal, or quasirandom activity across time (Alvarez and Squire, 1994; McClelland, 1994; McClelland and Goddard, 1996; McClelland et al., 1995; Squire and Knowlton, 1995; Squire et al., 1989; Treves and Rolls, 1994) or during sleep (Buzsaki, 1996; Maquet et al., 1996; McClelland et al., 1995; Skaggs and M cNaughton, 1996; Wilson and McNaughton, 1994). I nterestingly, it has been shown in artificial neural network models that memory consolidation can occur as a consequence of repeated reactivations of memory representations (Alvarez and Squire, 1994) or rehearsal and pseudorehearsal processes (Robins, 1995, 1996) (see also the discussion in McClelland, 1994; McClelland and Goddard, 1996; McClelland et al., 1995). If this is the case then the repeated encoding and recall during practice should contribute to memory consol idation and as a consequence the neocortex should be able to support declarative retrieval, if not independently so at least less dependently on the interaction with the MTL. Our data are consistent with such an interpretation.

A third interpretation relates the changing role of the MTL as a consequence of repeated encoding to the difference between episodic and semantic memory. Semantic memory has been hypothesized to result from the accumulation of many memory episodes (Baddeley, 
1994) or information gathered in the course of specific experiences (Squire, 1986; Tulving, 1983). Possibly, repeated encoding and recall would transform initial episodic memory into more semantic-like memory and the changing role of the MTL may then be interpreted as indicating that episodic memory retrieval may be more dependent on the function of the MTL compared to semantic memory retrieval.

A fourth perspective relates to the concepts of retrieval attempt and retrieval success. Retrieval attempt refers to processes engaged in trying to retrieve information from memory, while retrieval success re fers to processes selectively engaged when retrieval attempt is successful. In our experiment, the subjects were more successful in retrieving the stored information in TR during the 5 min of recall time compared to NR. If this interpretation is correct, this would imply that retrieval attempt with low retrieval success is associated with higher activity in the MTL compared to retrieval attempt with high retrieval success. This interpretation may be consistent with findings of a positive correlation between retrieval success and MTL activity (Nyberg et al., 1996), since the amount of encoding was not as varied in that study as in ours. However, during scanning (it should be remembered that the PET methodology is most sensitive to changes in activation during the first $30 \mathrm{~s}$ of scanning) the subjects were generally successful in retrieving their stored information during both NR and TR, though the rate of retrieval success was somewhat higher during TR compared to NR.

The data could also be seen as being consistent with a different hypothesis of the role of the MTL which suggests that the hippocampal formation may subserve aspects of retrieval in service of novelty assessment (Tulving et al., 1996), or recency detection (Brown, 1990), even if it may have less to contribute to retrieval of highly familiar information. This would also be in line with electrophysiological evidence of changing responses in hippocampal neurons to novel compared to familiar stimuli (Rolls et al., 1993).

A final perspective relates to a well-known problem in the computational theory of learning systems, i.e., the serial learning problem or the stability-plasticity dilemma (Arbib, 1995; Carpenter and Grossberg, 1993; Grossberg, 1987; Hertz et al., 1991). The possibility of rapid and effective learning requires system adaptability or network plasticity. This brings instability into the system and poses a threat to the stability of the same. In artificial neural networks this has been called catastrophic interference (McClelland et al., 1995; McCloskey and Cohen, 1989; Ratcliff, 1990) or catastrophic forgetting (Robins, 1995, 1996). Computational considerations concerning parallel distributed processing suggest that rapid learning through synaptic modifications and relational binding in a MTL module, memory consolidation through interaction between a MTL module and neocortical modules, and final integrative memory storage in neocortical modules may be a solution to the serial learning problem in certain learning systems (Cohen and O'Reilly, 1996; McClelland and Goddard, 1996; McClelland et al., 1995; Squireand Alvarez, 1995). I n this context, memory consolidation gets a functional interpretation in terms of successful and effective integration of newly acquired information into existing long-term memory. Our findings are consistent with such views.

\section{CONCLUSIONS}

In conclusion, we present experimental evidence for a changing role of the MTL in the recall of abstract visuospatial designs as a result of repeated encoding and recall, i.e., the MTL was more activated during retrieval in the less practiced memory state compared to the well-practiced memory state, indicating a dynamic role of the MTL in retrieval during the learning process. We also demonstrate that the activation of the MTL decreases as the subjects learn to draw abstract designs from memory, indicating a changing role of the MTL during recall in the earlier stages of acquisition compared to the well-encoded declarative memory state.

\section{ACKN O WLEDGMENTS}

This study was supported by grants from the Swedish Medical Research Council, the Karolinska Institute, the Swedish Bank Tercentenary Foundation, and the Knut and Alice Wallenberg Foundation. The help from Ellenor Andersson, P. H. Ghatan, Walter Pulka, Monica Serrander, Peter Söderholm, and Lennart Thurfjell is gratefully acknowledged.

\section{REFERENCES}

Alvarez, P., and Squire, L. R. 1994. Memory consolidation and the medial temporal lobe: A simple network model. Proc. Natl. Acad. Sci. USA 91:7041-7045.

Amit, D. J . 1989. Modeling Brain Function: The World of Attractor Neural Networks. Cambridge Univ. Press, Cambridge, UK.

Amit, D. J . 1995. The Hebbian Paradigm reintegrated: Local reverberations as internal representations. Behav. Brain Sci. 18:617626.

Arbib, M. A. 1995. The Handbook of Brain Theory and Neural Networks. MIT Press, Cambridge, MA.

Baddeley, A. 1994. Working memory: The interface between memory and cognition. In Memory Systems 1994 (D. L. Schacter and E. Tulving, Eds.), pp. 351-367. MIT Press, Cambridge, MA.

Baddeley, A. 1995. Working memory. In The Cognitive Neurosciences (M. S. Gazzaniga, Ed.), pp. 755-764. MIT Press, Cambridge, MA.

Beck, C., and Schlögl, F. 1993. Thermodynamics of Chaotic Systems: An Introduction. Cambridge Univ. Press, Cambridge, UK.

Berridge, M. S., Cassidy, E. H., and Terris, A. H. 1990. A routine, automated synthesis of oxygen-15-labeled butanol for positron tomography. J . Nucl. Med. 31:1727-1731.

Bibbig, A., Wennekers, T., and Palm, G. 1995. A neural network model 
of the cortico-hippocampal interplay and the representation of contexts. Behav. Brain Res. 66:169-175.

Brashers-Krug, T., Shadmehr, R., and Bizzi, E. 1996. Consolidation in human motor memory. Nature 382:252-255.

Brown, M. W. 1990. Why does the cortex have a hippocampus? In Learning and Computational Neuroscience: Foundations of Adaptive Networks (M. Gabriel and J . Moore, Eds.), pp. 233-282. MIT Press, Cambridge, MA.

Buzsaki, G. 1996. The hippocampo-neocortical dialogue. Cereb. Cortex 6:81-92.

Cahusac, P. B. M., Rolls, E., Miyashita, Y., and Niki, H. 1993. Modification of the response of hippocampal neurons in the monkey during the learning of a conditional spatial response task. Hippocampus 3:29-42.

Carpenter, G. A., and Grossberg, S. 1993. Normal and amnesic learning, recognition and memory by a neural model of corticohippocampal interaction. Trends Neurosci. 16:131-137.

Cohen, J. D., and O'Reilly, R. C. 1996. A preliminary theory of the interactions between prefrontal cortex and hippocampus that contribute to planning and prospective memory. In Prospective Memory: Theory and Applications (M. Brandimonte, G. O. Einstein, and M. A. McDaniel, Eds.). Erlbaum, Hillsdale, NJ .

Damasio, A. R., and Damasio, H. 1994. Cortical systems for retrieval of concrete knowledge: The convergence zone framework. In LargeScale Neuronal Theories of the Brain (C. Koch and J . L. Davies, Eds.), pp. 61-74. MIT Press, Cambridge, MA.

Eichenbaum, H. 1994. The hippocampal system and declarative memory in humans and animals: Experimental analysis and historical origins. In Memory Systems 1994 (D. L. Schacter and E. Tulving, Eds.), pp. 147-202. MIT Press, Cambridge, MA.

Friston, K. J ., Ashburner, J ., Frith, C. D., Poline, J . B., Heather, J . D., and Frackowiak, R. S. J . 1995a. Spatial registration and normalization of images. Hum. Brain Map. 3:165-189.

Friston, K. J ., Frith, C. D., and Passingham, R. E. 1992. Motor practice and neurophysiological adaptation in the cerebellum: A positron emission tomographic study. Proc. R. Soc. London 248:223228.

Friston, K. J ., Holmes, A. P., Worsley, K. J., Poline, J .-P., and Frackowiak, R. S. J . 1995b. Statistical parametric maps in functional imaging: A general linear approach. Hum. Brain Map. 2:189-210.

Fuster, J. M. 1994. Memory in the Cerebral Cortex: An Empirical Approach to Neural Networks in the Human and Non-human Primate MIT Press, Cambridge, MA.

Grady, C. L., Mcl ntosh, A. R., Horwitz, B., Maisog, J . M., Ungerleider, L. G., Mentis, M. J ., Pietrini, P., Schapiro, M. B., and Haxby, J . V. 1995. Age-related reductions in human recognition memory due to impaired encoding. Science 269:218-221.

Grafton, S. T., Hazeltine, E., and I vry, R. 1995. Functional mapping of sequence learning in normal humans. J . Cognit. Neurosci. 7:497510.

Grafton, S. T., Mazziotta, J . C., Presty, S., Friston, K. J ., Frackowiak, R. S. J ., and Phelps, M. E. 1992. Functional anatomy of human procedural learning determined with regional cerebral blood flow and PET. J . Neurosci. 12:2542-2548.

Grasby, P. M., Frith, C. D., Friston, K. J ., Bench, C., Frackowiak, R. S., and Dolan, R. J . 1993. Functional mapping of brain areas implicated in auditory-verbal memory function. Brain 116:1-20.

Greitz, T., Bohm, C., Holte, S., and Eriksson, L. 1991. A computerized brain atlas: Construction, anatomical content and some applications. J . Comput. Assisted Tomogr. 15:26-38.

Grossberg, S. 1987. Competitive learning: From interactive activation to adaptive resonance. Cognit. Sci. 11:23-63.

Haxby, J . V., Ungerleider, L. G., Horwitz, B., Maisog, J . M., Rapoport,
S. I., and Grady, C. L. 1996. Face encoding and recognition in the human brain. Proc. Natl. Acad. Sci. USA 93:922-927.

Haykin, S. 1994. Neural Networks: A Comprehensive Foundation. Macmillan Collage, New York.

Hertz, J ., Krogh, A., and Palmer, R. G. 1991. Introduction to the theory of neural computation. Addison-Wesley, Reading, MA. [Lecture notes: volumes of the Santa Fe studies in the sciences of complexity]

Ingvar, M., Eriksson, L., Greitz, T., Stone-Elander, S., Dahlbom, M., Rosenqvist, G., af Trampe, P., and von Euler, C. 1994. Methodological aspects of brain activation studies: Cerebral blood flow determined with $\left[{ }^{15} \mathrm{O}\right]$ butanol and positron emission tomography. J . Cereb. Blood Flow Metab. 14:628-638.

Insausti, R., Amaral, D. G., and Cowan, W. M. 1987a. The entorhinal cortex of the monkey. II. Cortical afferents. J. Comp. Neurol. 264:356-395.

Insausti, R., Amaral, D. G., and Cowan, W. M. 1987b. The entorhinal cortex of the monkey. III. Subcortical afferents. J . Comp. Neurol. 264:396-408.

J enkins, I. H., Brooks, D. J ., Nixon, P. D., Frackowiak, R. S., and Passingham, R. E. 1994. Motor sequence learning: A study with positron emission tomography. J . Neurosci. 14:3775-3790.

J ones-Gotman, M. 1986. Right hippocampal excision impairs learning and recall of a list of abstract designs. Neuropsychologia 24:659-670.

Kapur, N., Friston, K. J ., Young, A., Frith, C. D., and Frackowiak. 1995. Activation of human hippocampal formation during memory for faces: A PET study. Cortex 31:99-108.

Maquet, P., Peters, J .-M., Aerts, J ., Delfiore, G., Degueldre, C., Luxen, A., and Franck, G. 1996. Functional neuroanatomy of human rapid-eye-movement sleep and dreaming. Nature 383:163-166.

Markowitsch, H. J . 1995. Anatomical basis of memory disorders. In The Cognitive Neurosciences (M. S. Gazzaniga, Ed.), pp. 765-779. MIT Press, Cambridge, MA.

McClelland, J. L. 1994. The organization of memory: A parallel distributed processing approach. Rev. Neurol . (Paris) 150:570-579.

McClelland, J . L., and Goddard, N. H. 1996. Considerations arising from complementary learning systems: Perspective on hippocampus and neocortex. Hippocampus 6:654-665.

McClelland, J . L., McNaughton, B. L., and O'Reilly, R. C. 1995. Why there are complementary learning systems in the hippocampus and neocortex: Insights from the successes and failures of connectionist models of learning and memory. Psychol. Rev. 102:419-457.

McCloskey, M., and Cohen, N. J . 1989. Catastrophic interference in connectionist networks: The sequential learning problem. In The Psychology of Learning and Motivation: Advances in Research and Theory (G. H. Bower, Ed.). Academic Press, San Diego.

Mesulam, M. M. 1990. Large-scale neurocognitive networks and distributed processing for attention, language, and memory. Ann. Neurol. 28:597-613.

Nadel, L. 1994. Multiple memory systems: What and why, an update. In Memory Systems 1994 (D. L. Schacter and E. Tulving, Eds.), pp. 39-64. MIT Press, Cambridge, MA.

Nyberg, L., Mcl ntosh, A. R., Houle, S., Nilsson, L. G., and Tulving, E. 1996. Activation of medial temporal structures during episodic memory retrieval. Nature 380:715-717.

Oldfield, R. C., 1971. The assessment and analysis of handedness: The Edinburgh I nventory. Neuropsychologia 9:97-113.

O'Reilly, R. C., and McClelland, J . L. 1994. Hippocampal conjunctive encoding, storage, and recall: Avoiding a tradeoff. Hippocampus 6:661-682.

Raczkowski, D., Kalat, J. W., and Nebes, R. 1974. Reliability and validity of some handedness questionnaire items. Neuropsychol ogy 6:43-47. 
Raichle, M. E., Fiez, J . A., Videen, T. O., MacLeod, A.-M. K., Pardo, J . V., Fox, P. T., and Petersen, S. E. 1994. Practice-related changes in human brain functional anatomy during nonmotor learning. Cereb. Cortex 4:8-26.

Ratcliff, R. 1990. Connectionist models of recognition memory: Constraints imposed by learning and forgetting functions. Psychol. Rev. 97:285-308.

Robins, A. 1995. Catastrophic forgetting, rehearsal, and pseudorehearsal. Connect. Sci. 7:123-146.

Robins, A. 1996. Consolidation in neural networks and in the sleeping brain. Connect. Sci. 8:259-275.

Rolls, E. T., Cahusac, P. M., Feigenbaum, J. D., and Miyashita, Y. 1993. Responses of single neurons in the hippocampus of the macaque related to recognition memory. Exp. Brain Res. 93:299306.

Rugg, M. D., Fletcher, P. C., Frith, C. D., Frackowiak, R. S. J ., and Dolan, R. J . 1996. Differential activations of the prefrontal cortex in successful and unsuccessful memory retreival. Brain 119:20732083.

Rumelhart, D., and McClelland, J . 1986. Paralle Distributed Processing: Explorations in the Microstructures of Cognition. MIT Press, Cambridge, MA.

Schacter, D. L., Alpert, N. M., Savage, C. R., Rauch, S. L., and Albert, M. S. 1996. Conscious recollection and the human hippocampal formation-Evidence from positron emission tomography. Proc. Natl. Acad. Sci. USA 93:321-325.

Schacter, D. L., and Tulving, E. 1994. What are the memory systems of 1994? In Memory Systems 1994 (E. Tulving and D. L. Schacter, Eds.), pp. 1-38. MIT Press, Cambridge, MA.

Shapiro, M. L., and Olton, D. S. 1994. Hippocampal function and interference. In Memory Systems 1994 (D. L. Schacter and E. Tulving, Eds.), pp. 87-118. MIT Press, Cambridge, MA.

Singer, W., and Gray, C. M. 1995. Visual feature integration and the temporal correlation hypothesis. Annu. Rev. Neurosci. 18:555-586.

Skaggs, W. E., and McNaughton, B. L. 1996. Replay of neuronal firing sequences in rat hippocampus during sleep following spatial experience. Science 271:1870-1873.

Squire, L. R. 1986. Mechanisms of memory. Science 232:1612-1619.

Squire, L. R. 1992. Memory and the hippocampus: A synthesis from findings with rats, monkeys, and humans. Psychol. Rev. 99:195231.

Squire, L. R. 1994. Declarative and nondeclarative memory: Multiple brain systems supporting learning and memory. In Memory Sys- tems 1994 (D. L. Schacter and E. Tulving, Eds.), pp. 203-231. MIT Press, Cambridge, MA.

Squire, L. R., and Alvarez, P. 1995. Retrograde amnesia and memory consolidation: A neurobiological perspective. Curr. Opin. Neurobiol. 5:169-177.

Squire, L. R., Knowlton, B., and Musen, G. 1993. The structure and organization of memory. Annu. Rev. Psychol. 44:453-495.

Squire, L. R., and Knowlton, B. J . 1995. Memory, hippocampus and brain systems. In The Cognitive Neurosciences (M. S. Gazzaniga, Ed.), pp. 825-837. MIT Press, Cambridge, MA.

Squire, L. R., Shimamura, A. P., and Amaral, D. G. 1989. Memory and the hippocampus. In Neuronal Models of Plasticity: Experimental and Theoretical Approaches (J . H. Byrneand W. O. Berry, Eds.), pp. 209-239. Academic Press, San Diego.

Squire, L. R., and Zola-Morgan, S. 1991. The medial temporal Iobe memory system. Science 253:1380-1386.

Suzuki, W. A., and Amaral, D. G. 1994. Perirhinal and parahippocampal cortices of the macaque monkey: Cortical afferents. J . Comp. Neurol. 350:497-533.

Talairach, J ., and Tournoux, P. 1988. Co-planar Stereotaxic Atlas of theHuman Brain. Thieme Verlag, Stuttgart.

Treves, A., and Rolls, E. T. 1994. Computational analysis of the role of the hippocampus in memory. Hippocampus 4:374-391.

Tulving, E. 1983. Elements of Episodic Memory. Clarendon, Oxford, UK.

Tulving, E. 1995. Organization of memory: Quo vadis? In The Cognitive Neurosciences (M. S. Gazzaniga, Ed.), pp. 839-847. MIT Press, Cambridge, MA.

Tulving, E., Markowitsch, H. J ., Craik, F. I. M., Habib, R., and Houle, S. 1996. Novelty and familiarity activations in PET studies of memory encoding and retrieval. Cereb. Cortex 6:71-79.

Wienhard, K., Dahlbom, M., Eriksson, L., Michel, C., Bruckbauer, T., Pietrzyk, U., and Heiss, W.-D. 1994. The ECAT EXACT HR: Performance of a new high resolution positron scanner. J . Comput. Assisted Tomogr. 18:110-118.

Wilson, M. A., and McNaughton, B. L. 1994. Reactivation of hippocampal ensemble memories during sleep. Science 265:676-679.

Worsley, K. J ., Evans, A. C., Marrett, S., and Neelin, P. 1992. A three-dimensional statistical analysis for CBF activation studies in human brain. J . Cereb. Blood Flow Metab. 12: 900-918.

Zola-Morgan, S., and Squire, L. R. 1993. Neuroanatomy of memory. Annu. Rev. Neurosci. 16:547-563.

Zola-Morgan, S. M., and Squire, L. R. 1990. The primate hippocampal formation: Evidence for a time-limited role in memory storage. Science 250:288-290. 